\title{
Article \\ BolTLP1, a Thaumatin-like Protein Gene, Confers Tolerance to Salt and Drought Stresses in Broccoli (Brassica oleracea L. var. Italica)
}

\author{
Lixia He ${ }^{1,+}$, Lihong Li ${ }^{1,+}$, Yinxia Zhu ${ }^{1}$, Yu Pan ${ }^{1}$, Xiuwen Zhang ${ }^{1}$, Xue Han ${ }^{1}$, Muzi Li ${ }^{2}$, Chengbin Chen $^{1}$, \\ Hui Li ${ }^{2, *}$ and Chunguo Wang ${ }^{1,3,4, *}$
}

1 Department of Genetics and Cell Biology, College of Life Sciences, Nankai University, Tianjin 300071, China; helx@mail.nankai.edu.cn (L.H.); 1lh348536673@126.com (L.L.); 18322712771@163.com (Y.Z.); Pan1997666@126.com (Y.P.); zxw20150316@163.com (X.Z.); hx18322106151@126.com (X.H.); chencb@nankai.edu.cn (C.C.)

2 College of Horticulture and Landscape, Tianjin Agricultural University, Tianjin 300384, China; mz5872991001@163.com

3 State Key Laboratory of Tree Genetics and Breeding, Northeast Forestry University, Harbin 150040, China

4 State Key Laboratory of Vegetable Germplasm Innovation, Tianjin Academy of Agricultural Sciences, Tianjin 300381, China

* Correspondence: lihui@tjau.edu.cn (H.L.); wangcg@nankai.edu.cn (C.W.)

+ Lixia He and Lihong Li contributed equally to this work.

\section{check for} updates

Citation: He, L.; Li, L.; Zhu, Y.; Pan, Y.; Zhang, X.; Han, X.; Li, M.; Chen, C.; Li, H.; Wang, C. BolTLP1, a Thaumatin-like Protein Gene, Confers Tolerance to Salt and Drought Stresses in Broccoli (Brassica oleracea L. var. Italica). Int. J. Mol. Sci. 2021, 22, 11132. https://doi.org/10.3390/ ijms222011132

Academic Editor: Juan Manuel Ruiz Lozano

Received: 2 September 2021

Accepted: 11 October 2021

Published: 15 October 2021

Publisher's Note: MDPI stays neutral with regard to jurisdictional claims in published maps and institutional affiliations.

Copyright: (c) 2021 by the authors. Licensee MDPI, Basel, Switzerland. This article is an open access article distributed under the terms and conditions of the Creative Commons Attribution (CC BY) license (https:// creativecommons.org/licenses/by/ $4.0 /)$.

\begin{abstract}
Plant thaumatin-like proteins (TLPs) play pleiotropic roles in defending against biotic and abiotic stresses. However, the functions of TLPs in broccoli, which is one of the major vegetables among the B. oleracea varieties, remain largely unknown. In the present study, bolTLP1 was identified in broccoli, and displayed remarkably inducible expression patterns by abiotic stress. The ectopic overexpression of bolTLP1 conferred increased tolerance to high salt and drought conditions in Arabidopsis. Similarly, bolTLP1-overexpressing broccoli transgenic lines significantly improved tolerance to salt and drought stresses. These results demonstrated that bolTLP1 positively regulates drought and salt tolerance. Transcriptome data displayed that bolTLP1 may function by regulating phytohormone (ABA, ethylene and auxin)-mediated signaling pathways, hydrolase and oxidoreductase activity, sulfur compound synthesis, and the differential expression of histone variants. Further studies confirmed that RESPONSE TO DESICCATION 2 (RD2), RESPONSIVE TO DEHYDRATION 22 (RD22), VASCULAR PLANT ONE-ZINC FINGER 2 (VOZ2), SM-LIKE 1B (LSM1B) and MALATE DEHYDROGENASE (MDH) physically interacted with bolTLP1, which implied that bolTLP1 could directly interact with these proteins to confer abiotic stress tolerance in broccoli. These findings provide new insights into the function and regulation of bolTLP1, and suggest potential applications for bolTLP1 in breeding broccoli and other crops with increased tolerance to salt and drought stresses.
\end{abstract}

Keywords: thaumatin-like proteins (TLPs); bolTLP1; broccoli; salt stress; drought stress

\section{Introduction}

Thaumatin-like proteins (TLPs) are widely distributed in plants, animals and fungi [1], and are named for their high sequence similarity to the thaumatin protein initially identified in shrub Thaumatococcus daniellii Benth [2]. Most TLPs contain about 200 amino acids and have a molecular mass ranging from 21 to $26 \mathrm{kDa}$, except the poplar TLPs, most of which are 24 to $34 \mathrm{kDa}$ in size $[1,3,4]$. In plants, TLPs are classified as pathogenesis-related protein family 5 (PR-5), one out of the 17 defense-related PR protein families [5,6]. Examination of genome databases revealed at least 37 TLPs in Oryza sativa, 33 in Zea mays, 18 in Vitis vinifera, 25 in Arabidopsis thaliana, 28 in Prunus persica and 42 in Populus trichocarpa [4,7]. However, the functions of the majority of TLPs are still unknown. Previous reports demonstrated that a few plant TLPs play important roles in defending against pathogen infection, particularly 
fungal infection. A $20 \mathrm{kDa}$ TLP from the French bean legumes displays considerable resistance against Fusarium oxysporum, Pleurotus ostreatus and Coprinus comatus [8]. In Cassia didymobotrya, a $23 \mathrm{kDa}$ TLP was identified, which exerts antifungal activity toward some Candida species [9]. TaLr35PR5, a TLP from wheat, was involved in Lr35-mediated adult wheat defense in response to leaf rust attack [10]. The recombinant expression of Mald2, a TLP identified in apple, conferred antifungal activity against Fusarium oxysporum and Penicillium expansum in Nicotiana benthamiana [11]. In tobacco, the ectopic overexpression of PpTLP from Pyrus pyrifolia or AdTLP from Arachis diogoi can enhance resistance to Rhizoctonia solani $[12,13]$. Transgenic canola, wheat and banana overexpressing TLPS from rice all exhibit increased resistance to diverse pathogens [14-16]. In addition to resistance against biotic stress, some TLPS in plants also respond to abiotic stress. Transgenic tobacco plants ectopically expressing AdTLP not only have enhanced resistance to Rhizoctonia solani, but also have improved tolerance to salt and oxidative stresses [13]. The ectopic expression of ObTLP1 isolated from Ocimum basilicum enhances tolerance to dehydration and salt stresses in Arabidopsis [17]. Under drought stress, TLPS showing inducible expression patterns were also identified in carrot and tea, suggesting that these TLPS could be involved in drought response $[18,19]$. In addition, TLPs have also been demonstrated to play roles in regulating plant growth and development including fruit maturation, lentinan degradation and seed development [20-24]. These investigations indicate that TLPS have undergone functional diversification in plant species, and play pleiotropic roles in response to biotic and abiotic stresses as well as in the regulation of growth and development [4,7].

Broccoli (Brassica oleracea L. var. italica) is one of the major vegetables among the Brassica oleracea varieties, and is widely planted in Asia, Europe and North America [25]. Soil salinization and drought are two potential threats in planting broccoli, which can result in curd yield reduction or even no harvest [26]. Breeding broccoli varieties with high tolerance to drought and salt is an important target for broccoli breeders. Several studies related to the responses to these two abiotic stresses were reported in broccoli and other species of Brassica. Broccoli pre-treating with SA and chitosan showed the highest drought stress recovery in a dose-dependent manner [27]. Drought-tolerant broccoli cultivars presented higher levels of methionine and ABA than that of the drought-sensitive cultivars [28]. 42 putative known and 39 putative candidate miRNAs displayed differentially expressed patterns between control and salt-stressed broccoli [29]. Bra-botrytis-ERF056 from cauliflower was demonstrated to increase tolerance to salt and drought stresses in overexpressing bra-botrytis-ERF056 Arabidopsis [30]. BrEXLB1 is involved in drought tolerance in Brassica rapa [31]. BnaABF2 from Brassica napus confers enhanced drought and salt tolerance in transgenic Arabidopsis [32]. BolOST1 was dramatically induced by drought and high-salt stress, and the ectopic expression of BolOST1 restored the drought-sensitive phenotype of ost1 [33]. However, natural response to salt and drought stresses in broccoli is still largely unknown. In our previous transcriptome data analysis, a TLP gene named bolTLP1 was identified in broccoli which exhibited a significant positive response to salt stress. However, the role of bolTLP1 is unknown. In the present study, the full-length cDNA of bolTLP1 was cloned, and its expression patterns under salt stress and drought stress were analyzed. Transgenic Arabidopsis and broccoli overexpressing bolTLP1 were generated. The phenotypes of these bolTLP1 overexpressing transgenic plants under salt and drought stresses were observed. The genes and regulatory processes involved in bolTLP1-mediated tolerance to abiotic stress were explored.

\section{Results}

\subsection{BolTLP1 Was Identified in Broccoli}

In previous comparative transcriptome sequence analysis to identify genes involved in salt stress response in broccoli, bolTLP1 was found to be rapidly induced under salt stress condition (Figure 1A). In the present study, the full-length cDNA of bolTLP1 was identified based on the ESTs of this gene from broccoli transcriptome data. Sequence analysis indicated that the coding region of bolTLP1comprises $696 \mathrm{bp}$ and encodes a predicted 
231-amino acid protein, which contained a conserved TLP domain. The phylogenetic analysis indicated that bolTLP1 and 1 out of the 29 TLP genes identified in Brassica oleracea var. oleracea (XP_013621285), are classified into a separate group, and they appear to show far genetic distance with other TLP genes (Figure 1B).
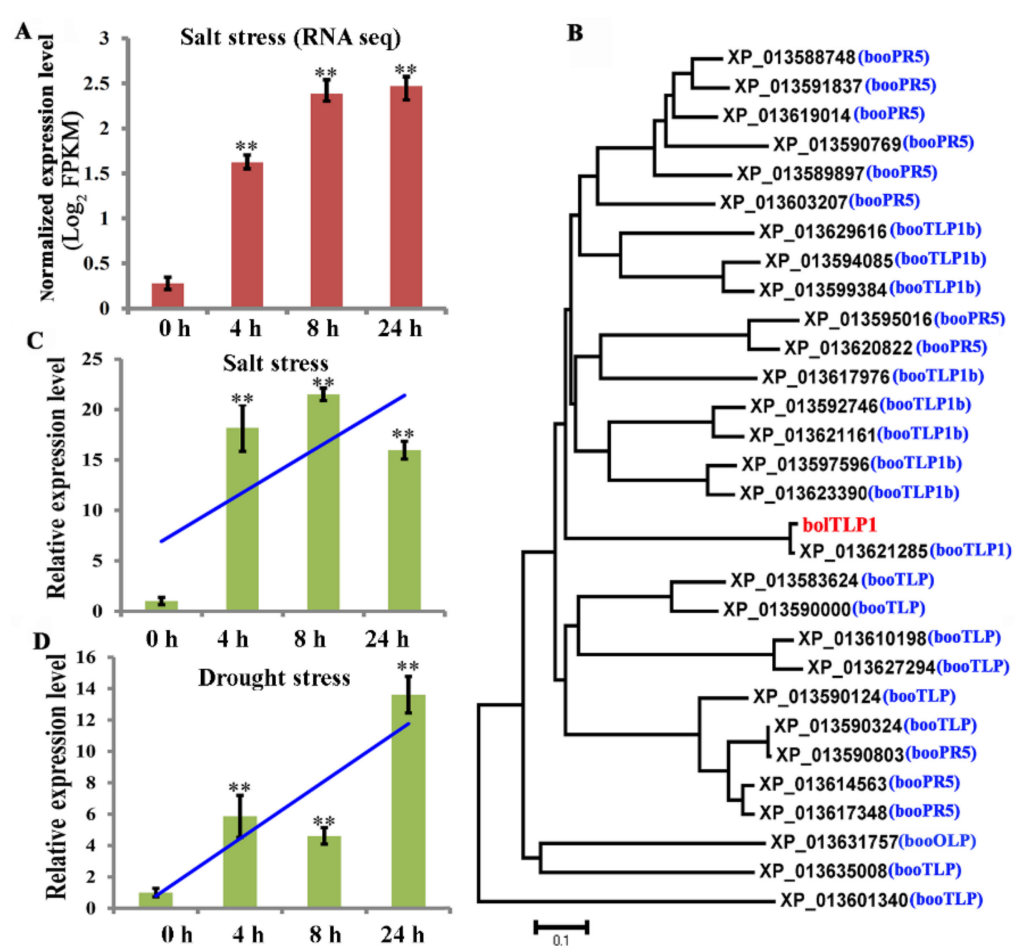

Figure 1. Phylogenetic tree and expression profiles of bolTLP1 under salt and drought stresses. (A): Expression profiles of bolTLP1 at $0 \mathrm{~h}$ (control), $4 \mathrm{~h}, 8 \mathrm{~h}$ and $24 \mathrm{~h}$ after salt stress $(200 \mathrm{mM} \mathrm{NaCl}$ treatment) detected by RNA-seq. (B): Phylogenetic analysis of bolTLP1 and genes with a TLP domain from Brassica oleracea var. oleracea (Bootstrap value $=1000$ ). The bar indicated the genetic distance of 0.1. The red font showed the position of bolTLP1. (C): Expression profiles of bolTLP1 at $0 \mathrm{~h}$ (control), $4 \mathrm{~h}, 8 \mathrm{~h}$ and $24 \mathrm{~h}$ after salt stress ( $200 \mathrm{mM} \mathrm{NaCl}$ treatment) detected by qRT-PCR. (D): Expression profiles of bolTLP1 at $0 \mathrm{~h}$ (control), $4 \mathrm{~h}, 8 \mathrm{~h}$ and $24 \mathrm{~h}$ after mimetic drought stress ( $300 \mathrm{mM}$ mannitol treatment). The blue lines indicated the expression trends of bolTLP1 under salt and drought stresses. ** indicated the significantly differential expression levels of bolTLP1 under salt and drought stresses $(p<0.01)$.

\subsection{BolTLP1 Positively Responded to Salt and Drought Stresses in Broccoli}

The expression pattern of bolTLP1 under salt treatment in broccoli was further confirmed by qRT-PCR. Consistent with the expression trend identified by comparative transcriptome sequence analysis, the expression level of bolTLP1 was increased significantly in $4 \mathrm{~h}$ after exposure to salt stress (Figure 1C). In addition, the expression pattern of bolTLP1 in the leaves of broccoli treated with $300 \mathrm{mM}$ mannitol, which could mimic drought condition, was explored. Similar to observations under salt stress, the expression level of bolTLP1 was significantly higher under drought stress than that observed under control condition $(0 \mathrm{~h})$ (Figure 1D).

\subsection{Overexpression of bolTLP1 Increased Resistance to Salt and Drought Stresses in Arabidopsis}

To further uncover the roles of bolTLP1, a transformation vector containing the bolTLP1 under the control of the enhanced CaMV 35S promoter was constructed, and used to transform Arabidopsis. A total of 26 independent 35S::bolTLP1 transgenic lines were obtained (Figure S1). Under normal growth conditions, there were no phenotypic differences between the 35S::bolTLP1 transgenic lines and the vector controls, thereby indicating that the overexpression of bolTLP1 did not affect the growth and development of the 
transgenic lines in Arabidopsis (Figure 2A). To determine if overexpression of bolTLP1 could improve resistance to salt and/or drought stress, 35S::bolTLP1 Arabidopsis lines were planted in soil, and the 25-day-old seedlings were subjected to 125 and $200 \mathrm{mM}$ $\mathrm{NaCl}$ treatments, respectively. At 12 days after $200 \mathrm{mM} \mathrm{NaCl}$ treatment, the aerial organs of the vector controls were wilted, growth was retarded, and leaves were yellow and senescent. Compared with the vector controls, the 35S::bolTLP1 Arabidopsis was only slightly affected by the high salt condition (Figure 2B). At 18 days after salt stress, the growth of the remaining vector control plants was substantially inhibited. The survival rate of 35S::bolTLP1 Arabidopsis was significantly higher than that of the vector controls, and the aerial organs of the 35S::bolTLP1 Arabidopsis still showed normal growth. (Figure 2C,G). Under $125 \mathrm{mM}$ NaCltreatment, although over $45 \%$ of the vector control plants died, almost all individual plants of the 35S::bolTLP1 Arabidopsis survived (Figure 2G). At both low (125 $\mathrm{mM})$ and high $(200 \mathrm{mM}) \mathrm{NaCl}$ treatments, the primary and lateral roots of the 35S::bolTLP1 Arabidopsis were stronger than those of the vector controls (Figure 2D-F).
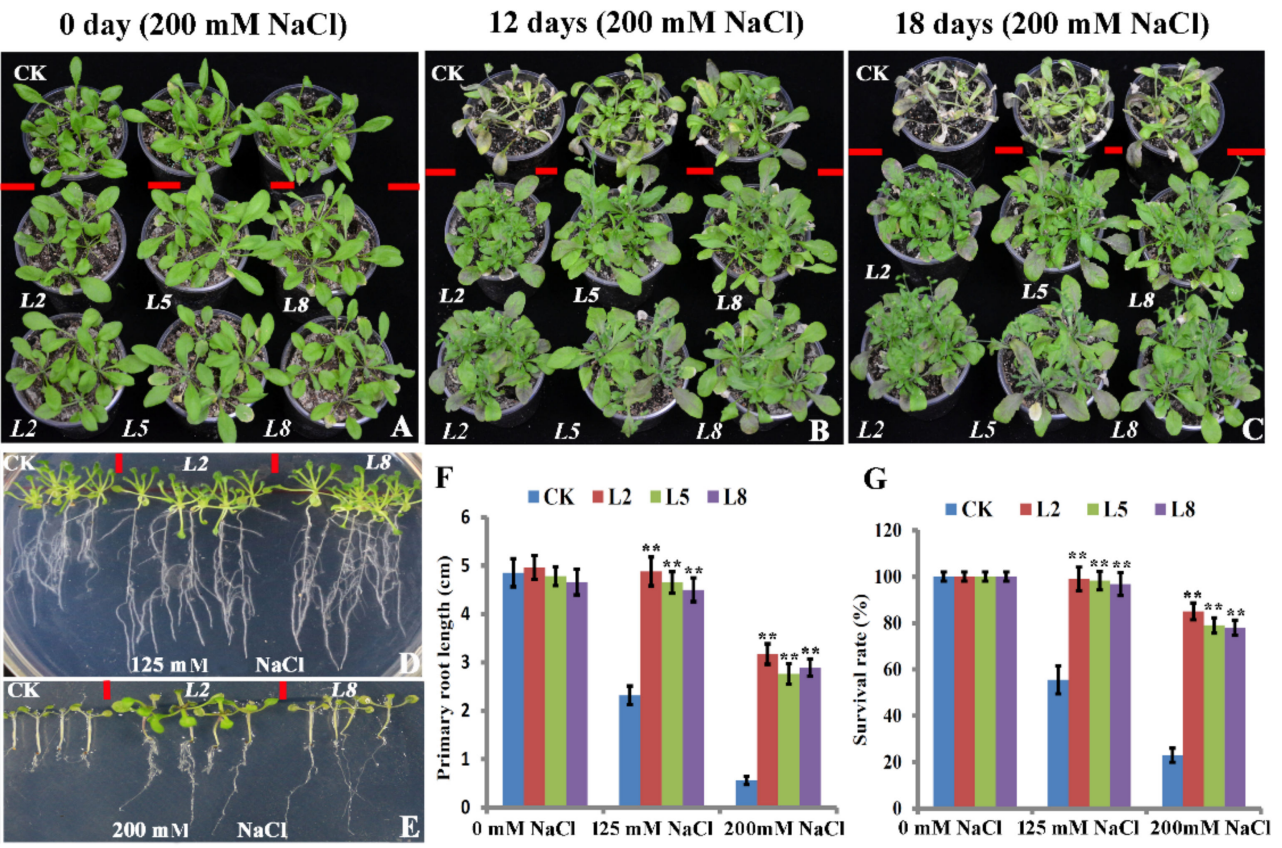

Figure 2. Phenotypes of transgenic Arabidopsis plants overexpressing bolTLP1 under salt stress. (A-C): Phenotypes of 35S::bolTLP1 Arabidopsis at 0 day, 12 days and 18 days after irrigated with $200 \mathrm{mM}$ $\mathrm{NaCl}$ in nutrient soil, respectively. (D,E): Phenotypes of 35S::bolTLP1 Arabidopsis seedlings at 15 days after growing in 1/2 MS medium with $125 \mathrm{mM}$ and $200 \mathrm{mM} \mathrm{NaCl}$, respectively. (F): Primary root length of 35S::bolTLP1 Arabidopsis at 15 days after growing in 1/2 MS medium with $125 \mathrm{mM}$ and $200 \mathrm{mM} \mathrm{NaCl}$. (G): Survival rates of 35S::bolTLP1 Arabidopsis at 18 days after irrigated with $125 \mathrm{mM}$ and $200 \mathrm{mM} \mathrm{NaCl}$ in nutrient soil. (Student's $t$-test, ${ }^{* *} p<0.01$; data are means $\pm \mathrm{SD}(n \geq 15)$ ). The top row of $(\mathrm{A}-\mathrm{C})$ showed the vector controls $(\mathrm{CK})$, and the middle and bottom rows showed the independent 35S::bolTLP1 Arabidopsis lines (L2, L5 and L8).

The 35S::bolTLP1 Arabidopsis plants were treated with water deficit. At 15 days after the water deficit, the leaves of the vector controls were seriously withered, and plant growth was inhibited. The growth and development of the 35S::bolTLP1 Arabidopsis were much better than those of the vector controls. In brief, the aerial organs of the 35S::bolTLP1 Arabidopsis were stronger than those of the vector controls, and their leaves were normal, although some of them also became senescent. The survival rate of 35S::bolTLP1 Arabidopsis was over $75 \%$, whereas only $10 \%$ of the vector control plants survived after rewatering (Figure 3A-C,G). To further confirm the roles of bolTLP1 under drought stress, the 35S::bolTLP1 Arabidopsis plants were treated with different concentrations of mannitol. At $200 \mathrm{mM}$ mannitol, the aerial organs of the 35S::bolTLP1 Arabidopsis were larger than 
those of the vector controls. Similarly, at $300 \mathrm{mM}$ mannitol, the growth and development of the aerial organs of the 35S::bolTLP1 Arabidopsis were still stronger than those of the vector controls (Figure 3D,E). Nevertheless, the roots, especially the primary roots, were not significantly affected by the mimic drought stresses (Figure 3F).

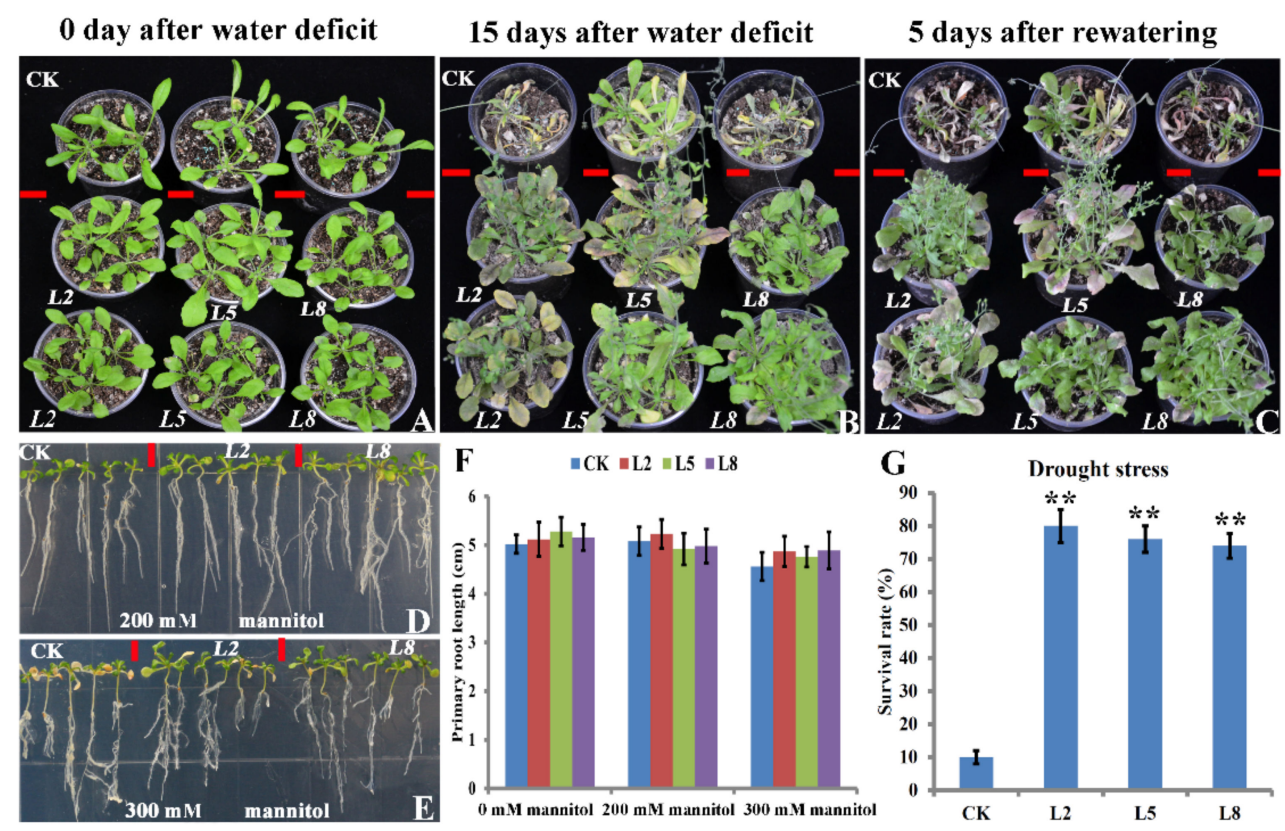

Figure 3. Phenotypes of transgenic Arabidopsis plants overexpressing bolTLP1 under drought stress. (A,B): Phenotypes of 35S::bolTLP1 Arabidopsis at 0 day and 15 days after the water deficit. (C): Phenotypes of 35S::bolTLP1 Arabidopsis at 5 days after rewatering. (D,E): Phenotypes of 14-day-old 35S::bolTLP1 Arabidopsis seedlings growing in 1/2 MS medium with $200 \mathrm{mM}$ and $300 \mathrm{mM}$ mannitol, respectively. (F): Primary root length of 35S::bolTLP1 transgenic Arabidopsis at 14 days after growing in 1/2 MS medium with $200 \mathrm{mM}$ and $300 \mathrm{mM}$ mannitol. (G): Survival rate of 35S::bolTLP1 Arabidopsis at 5 days after rewatering. (Student's $t$-test, ${ }^{* *} p<0.01$; data are means $\pm \operatorname{SD}(n \geq 20)$ ). The top row of $\mathrm{A}, \mathrm{B}$ and $\mathrm{C}$ showed the vector controls $(\mathrm{CK})$, and the middle and bottom rows showed the independent 35S::bolTLP1 Arabidopsis lines (L2, L5 and L8).

\subsection{Transgenic Broccoli Overexpressing bolTLP1 Exhibited High Salt and Drought Tolerance}

BolTLP1 was also overexpressed in broccoli. A total of 16 independent 35S::bolTLP1 broccoli transgenic lines were obtained (Figure S1). Similar to the 35S::bolTLP1 Arabidopsis, the growth and development of the 35S::bolTLP1 broccoli were unaffected under the normal growth conditions (Figure 4A). The 30-day-old 35S::bolTLP1 broccoli seedlings were irrigated with $200 \mathrm{mM} \mathrm{NaCl}$. At 13 days after the salt treatment, the growth of the 35S::bolTLP1 broccoli was normal. Under these conditions, the vector controls grew slower and the first leaves showed signs of senescence (Figure 4B). As time went on, the 35S::bolTLP1 broccoli showed slight salt damage, but their growth vigor was much better than that of the vector controls (Figure 4C-E). The growth and development of the 35S::bolTLP1 broccoli under drought stress were also examined. The phenotypic data demonstrated that at 12 days after the water deficit, the aerial organs of the vector controls became withered, and their first and second leaves were senescing. Interestingly, at this same stage of water deficit, the growth of the 35S::bolTLP1 broccoli was not considerably affected, and their aerial organs were larger and stronger than those of the vector controls. At 15 days after the water deficit, although the leaves of the 35S::bolTLP1 broccoli and vector controls both showed withering, the growth vigor of the vector controls was inhibited to a greater degree than the 35S::bolTLP1 broccoli. After rewatering, the majority of the 35S::bolTLP1 broccoli (over $78 \%$ ) survived, whereas almost all vector controls died (Figure $4 \mathrm{~F}-\mathrm{J}$ ). 

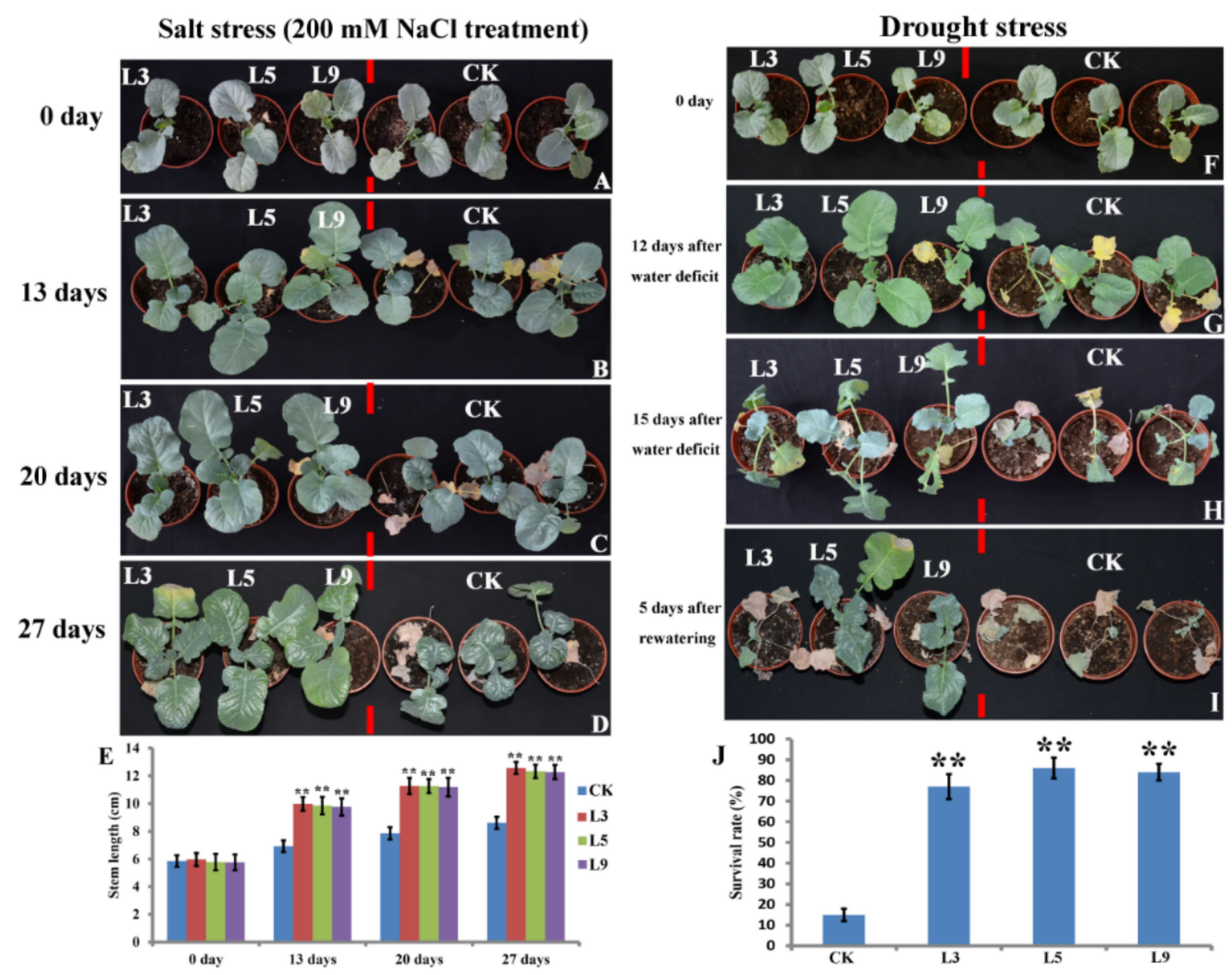

Figure 4. Phenotypes of transgenic broccoli plants overexpressing bolTLP1 under salt and drought stresses. (A-D): Phenotypes of 35S::bolTLP1 broccoli at 0 day, 13 days, 20 days and 27 days after irrigated with $200 \mathrm{mM} \mathrm{NaCl}$, respectively. (E): Stem length of 35S::bolTLP1 broccoli at 0 day, 13 days, 20 days and 27 days after irrigated with $200 \mathrm{mM} \mathrm{NaCl}$. (Student's $t$-test, ${ }^{* *} p<0.01$; data are means \pm $\mathrm{SD}(n \geq 18))$. (F-H): Phenotypes of 35S::bolTLP1 broccoli at 0 day, 12 days and 15 days after water deficit, respectively. (I): Phenotypes of 35S::bolTLP1 broccoli at 5 days after rewatering. (J): Survival rate of 35S::bolTLP1 broccoli at 5 days after rewatering. (Student's $t$-test, ${ }^{* *} p<0.01$; data are means \pm SD $(n \geq 15))$. L3, L5 and L9 indicated the three independent 35S::bolTLP1 broccoli lines. CK indicated the vector control plants.

\subsection{DEGs Were Confirmed between the 35S::bolTLP1 Broccoli and Vector Control}

Comparative transcriptome analysis was conducted to identify the DEGs between the 35S::bolTLP1 broccoli and the vector controls. In total, 34,258 genes showing transcriptional expression were detected, among which 3284 genes showed significantly differential expression levels (corrected $p$-value $<0.01$ ). The expression levels of 2202 out of the 3284 DEGs were up-regulated in the 35S::bolTLP1 broccoli, and the other approximately $33 \%$ of the DEGs displayed lower expression levels in the 35S::bolTLP1 broccoli compared to the vector control plants (Table S1). GO functional annotations indicated that these DEGs were mapped to 933 GO terms in the biological process, 154 GO terms in the cellular component and 343 GO terms in the molecular function, among which 33, 1 and 1 GO terms were significantly enriched in biological process, molecular function and cellular component, respectively (corrected $p$-value < 0.01) (Figure 5, Table S2). The significantly enriched GO terms in the biological process were mainly involved in stress responses, glycosinolate and sulfur compound synthetic and metabolic process, and peptidase, proteolysis and hydrolase activity. The GO terms involved in oxidoreductase activity and chromatin organization were significantly enriched in the molecular function and the cellular components, respectively (Figure 5, Table S2). 


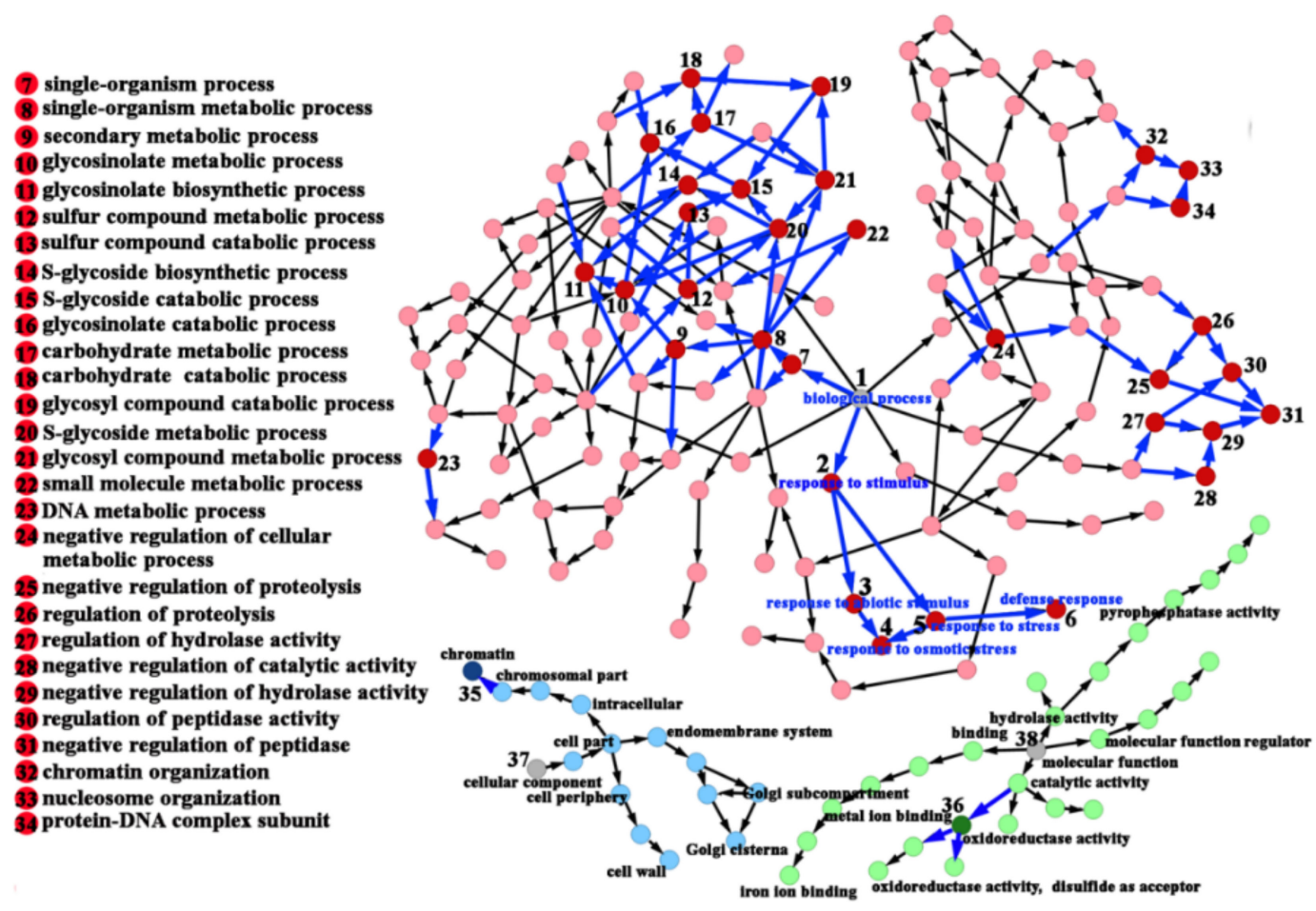

Figure 5. Regulatory network relationships of GO terms of the differentially expressed genes between 35S::bolTLP1 broccoli and the vector control plants. Each node represents a GO term. GO terms in biological process, cellular component and molecular function were distinguished by red, blue and green color, respectively. The significantly enriched GO terms (corrected $p$-value $<0.01$ ) are further marked by dark color, and their inclusion relations are highlighted by thick and blue arrows. The inclusion relations of other GO terms are marked by black arrows. Only those significantly enriched GO terms are serially numbered and their functional annotations were showed. The functional annotations of other enriched GO terms can be found in Table S2.

2.6. Expression Profiles of Genes Involved in ABA, Ethylene and Auxin-Mediated Signaling Pathways Were Significantly Altered by Overexpression of bolTLP1

According to the functional annotations of all DEGs by GO analysis, genes involved in the biotic and abiotic stress response processes were obviously predominant in the DEGs. At least 685 out of the 3284 DEGs (>21\%) were functionally associated with stress response processes. Among the stress response-associated DEGs, approximately 70\% of them displayed higher expression levels in the 35S::bolTLP1 broccoli than those in the vector controls (Table S3). A large proportion of these DEGs were key regulators in phytohormone-mediated signaling pathways. In brief, twenty-six DEGs were involved in the auxin signaling pathway. Twenty-four out of the 26 DEGs displayed higher expression levels in the 35S::bolTLP1 broccoli than those in the vector controls. Thirty-one genes involved in the ABA signaling pathway displayed differential expression patterns between the 35S::bolTLP1 broccoli and the vector controls, among which 14 genes, such as RD22, HVA22, RCI2A and ABSCISIC ACID-INSENSITIVE 5 (ABI 5), displayed significantly upregulated expression patterns in the 35S::bolTLP1 broccoli. At least 11 genes involved in the ethylene signaling pathway displayed differential expression patterns between the 35S::bolTLP1 broccoli and the vector controls (Figure 6A-C). The expression levels of some of these DEGs were further verified by qRT-PCR (Figure 7). 


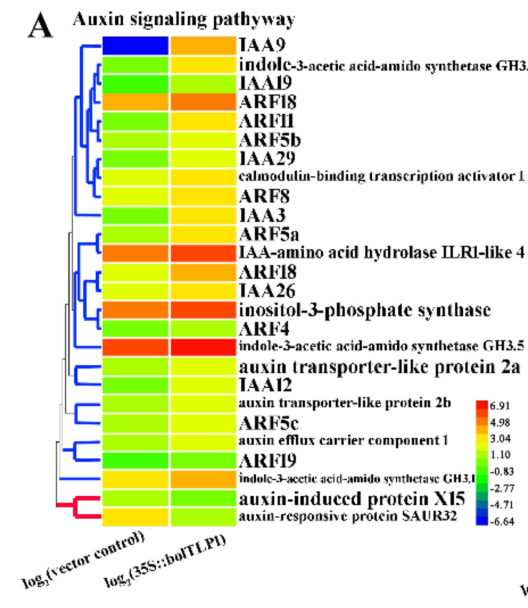

D Sulfur compound synthesis

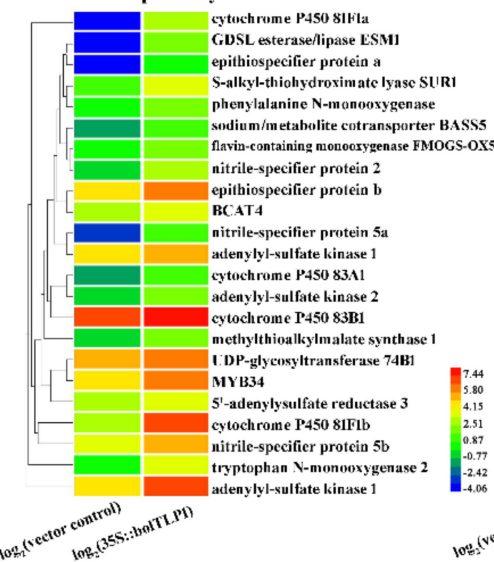

B $\mathrm{ABA}$ signaling pathyway

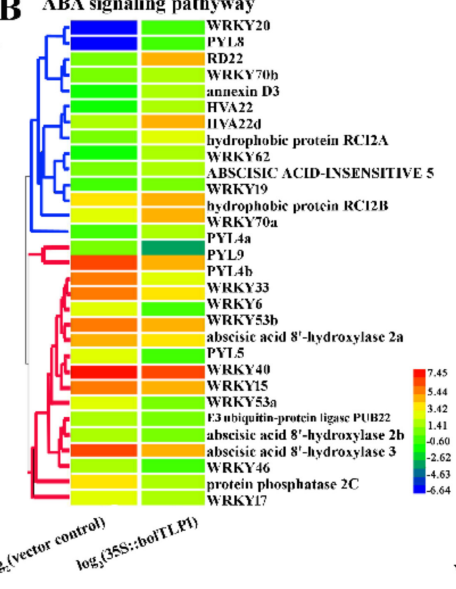

C Ethylene aignaling pathyway

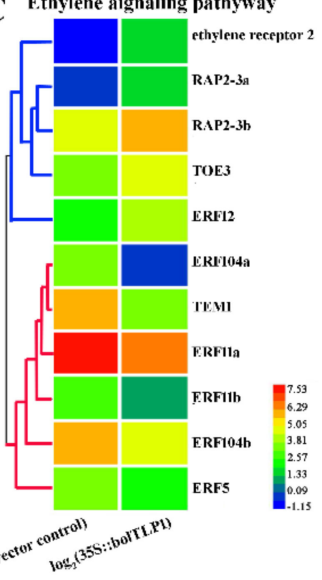

E Protease inhibitors

F Histone variants

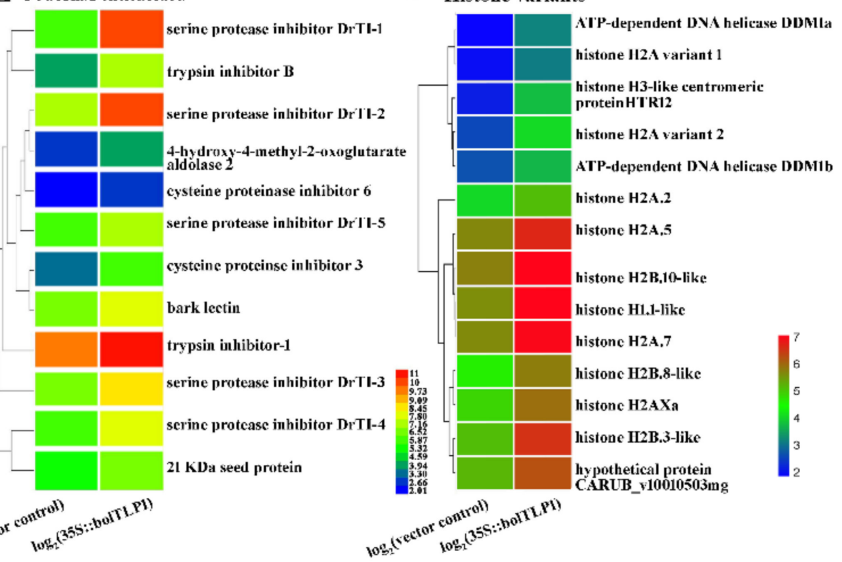

Figure 6. Transcriptional expression patterns of the differentially expressed genes involved in phytohormone-mediated signaling pathways (A-C), sulfur compound synthesis (D), protease inhibitors $(\mathbf{E})$ and histone variants $(\mathbf{F})$. The blue and red branches in $(\mathbf{A}-\mathbf{C})$ indicated the genes displayed significantly up-regulated and down-regulated expression in the 35S::bolTLP1 broccoli compared with the vector control (corrected $p$-value < 0.01), respectively. $\log _{2}$ (35S::bolTLP1) and $\log _{2}$ (vector control) indicated the $\log _{2}$ FPKM of bolTLP1 in 35S::bolTLP1 broccoli and vector control plants, respectively, which represents the normalized expression level of bolTLP1 detected by RNA-seq.

2.7. Genes Involved in Sulfur Compound Synthesis and Hydrolase/Oxidoreductase Activity Were Significantly Inductively Expressed by the Overexpression of bolTLP1

Besides the DEGs involved in stress response, at least 62 DEGs were confirmed to map the GO terms associated with the glycosinolate and sulfur compound synthesis and metabolism, which displayed significant enrichment in the biological process. Forty-seven out of the 62 DEGs, such as genes encoding cytochrome P450 81F1, epithiospecifier protein, S-alkyl-thiohydroximate lyase, adenylyl-sulfate kinase, nitrile-specifier protein, flavincontaining monooxygenase, 5'-adenylylsulfate reductase and cytochrome P450 83B1, had significantly higher expression levels in the 35S::bolTLP1 broccoli compared to the vector control plants. The majority of these genes play crucial roles in sulfur compound synthesis and metabolism (Figure 6D, Table S3). 


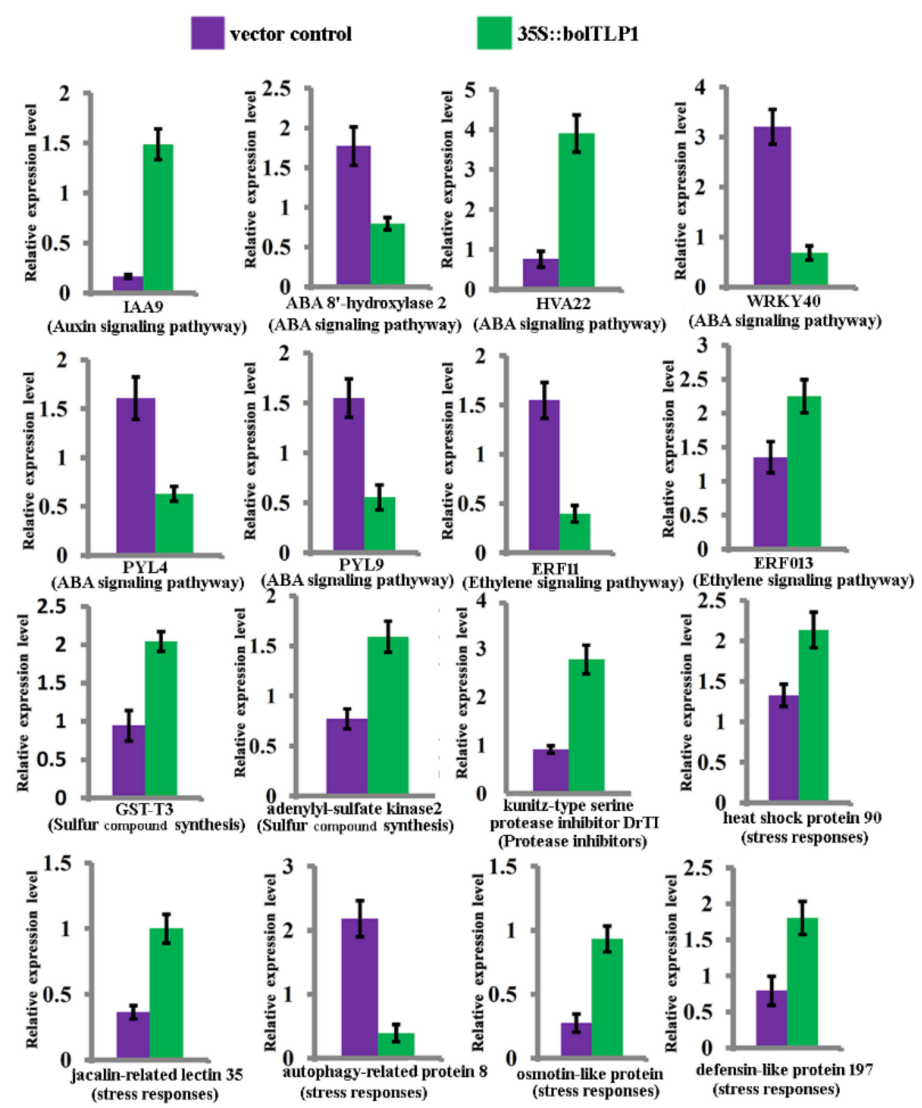

Figure 7. Identification of the relative expression levels of the representative genes by qRT-PCR. GST: glutathione S-transferase. PYL: abscisic acid receptor PYR/PYL/RCAR. ERF: ethylene-responsive transcription factor. IAA9: indol-yl-3-acetic acid 9. RD22: dehydration-responsive protein 22. 35S::bolTLP1: 35S::bolTLP1 broccoli. Vector control: vector control plants.

The expression patterns of genes related to significantly enriched GO terms involved in the activity of diverse enzymes, such as the proteolysis, peptidase, hydrolase and oxidoreductase, were also analyzed. A total of 12 genes including genes encoding kunitz-type serine protease inhibitor, cysteine proteinase inhibitor, trypsin inhibitor and 4-hydroxy4-methyl-2-oxoglutarate aldolase were associated with the activity of proteolysis and hydrolase, all of which exhibited significantly increased expression levels in the 35S::bolTLP1 broccoli (Figure 6E). At least 192 DEGs were confirmed to be associated with the oxidoreductase activity. The majority of them (146 out of 192) also exhibited higher expression levels in the 35S::bolTLP1 broccoli than those in the vector controls (Table S3). Moreover, 15 genes associated with the significantly enriched GO terms involved in chromatin organization, especially histone variants, were identified. All these genes showed increased expression levels in the 35S::bolTLP1 broccoli (Figure 6F, Table S3).

\subsection{Five Proteins Involved in Abiotic Stress Responses Were Confirmed to Interact with bolTLP1}

Yeast two-hybrid screening was conducted to identify proteins that could interact with bolTLP1. In total, 266 positive clones were identified and sequenced. These sequences were annotated as 20 diverse genes, among which RD2, RD22, VOZ2, LSM1B and MDH were functionally associated with stress responses in plants. The interaction of these five proteins with bolTLP1 was further confirmed by using bolTLP1 as the prey and RD2, RD22, VOZ2, LSM1B and MDH as the baits in the yeast two-hybrid assay (Figure 8A, Table S4). Quantitative expression analysis revealed that the expression levels of these stress response-associated genes were significantly higher in 35S::bolTLP1 broccoli than those in the vector controls (Figure 8B). 
A
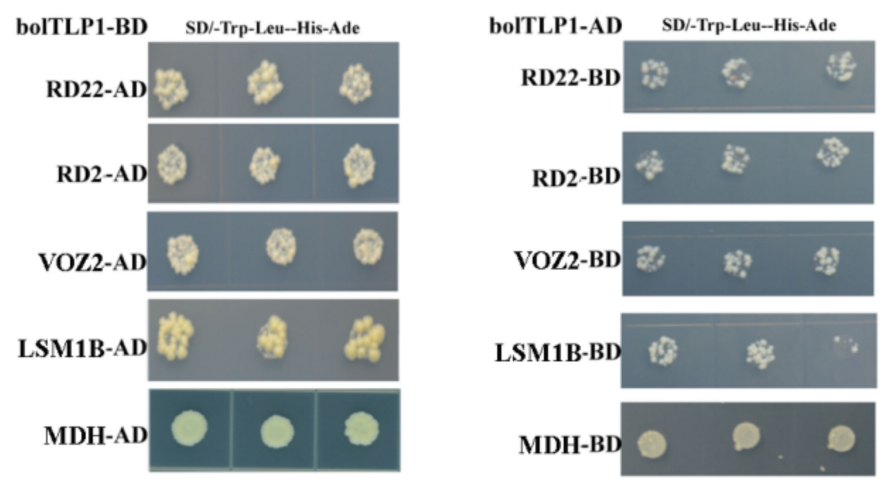

B

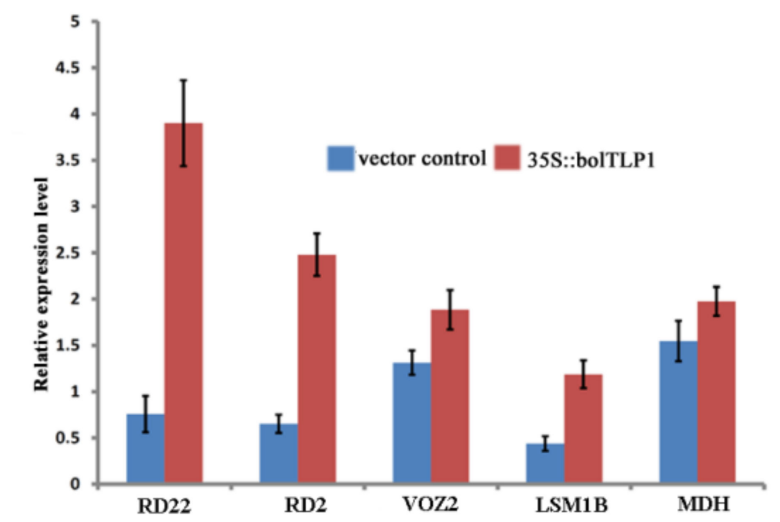

Figure 8. Identification and expression profiles of proteins interacting with bolTLP1. (A): Identification of proteins interacting with bolTLP1 by yeast two-hybrid assay. Three spots from left to right in each row indicated the 1:10 gradient dilution of yeast clone. (B): Relative expression levels of the five candidate genes, which encode proteins that interact with bolTLP1, in 35S::bolTLP1 broccoli (35S::bolTLP1) and the vector control plants (vector control).

\section{Discussion}

TLPS are present as a large group of PR-5 gene family in plants, and most TLPs were demonstrated to play a role in defending against pathogen $[1,9,11,14,16]$. Only a few TLPS have been demonstrated to function in response to abiotic stress. Nevertheless, in the present study, bolTLP1, a member of TLP subfamily, was first identified in broccoli. The expression profiles assay confirmed that bolTLP1 displayed inducible expression patterns under salt and drought stresses. Overexpression of bolTLP1 in broccoli, as well as in Arabidopsis, significantly increased the salt and drought tolerance of transgenic plants. These findings demonstrated that bolTLP1 is an important positive response factor that plays a role in combatting salt and drought stress in broccoli, and suggested the potential applications of bolTLP1 in breeding crops with high salt and drought tolerance via genetic engineering.

The molecular mechanisms by which the TLPs respond to abiotic stress remain largely unknown, although investigations have confirmed the roles of several TLP genes in cold temperature, salt and drought stresses $[13,17-19]$. In the present study, comparative transcriptome analysis revealed that the DEGs detected between the 35S::bolTLP1 broccoli and the vector controls were mainly involved in the regulatory processes including "stress responses", "glycosinolate and sulfur compound metabolic process", "peptidase, proteolysis and hydrolase activity", "oxidoreductase activity" and "chromatin organization" (Figure 5, Table S2). These results suggested that these regulatory processes should participate in bolTLP1-mediated tolerance to salt and drought stresses. Furthermore, the functional annotation analysis of the DEGs enriched in these regulatory processes confirmed that over $21 \%$ DEGs were involved in stress response processes, which represented the largest gene group in the DEGs. Remarkably, a large proportion of these DEGs were associated with phytohormone-mediated signaling pathways. For example, RD2, RD22, 
ABI5, WRKY15/17/20/33/40/46/53/62/70, abscisic acid 8'-hydroxylase 2, annexin, PUB23, HVA22, RCI2A, RCI2B and PYL4/5/8/9 are the important members of ABA-mediated signaling pathway, which all displayed significantly differential expression levels between the 35S::bolTLP1 broccoli and the vector controls (Figure 6B). Most of these genes were confirmed to play crucial roles in response to abiotic stresses in diverse plant species [34-38]. Interestingly, RD2 and RD22 were confirmed to directly interact with bolTLP1 (Figure 8). $R D 2$ and $R D 22$ are up-regulated by drought stress, salinity stress and exogenously supplied ABA. Their inducible expression has been used as a marker for abiotic stress $[39,40]$. In addition, at least 26 DEGs, such as auxin response factor $(A R F)$ 4/5/8/11/14/18/19, auxin transporter 2, IAA3/9/12/19/26/29, inositol-3-phosphate synthase, indole-3-aceticacidamido synthetase GH3, calmodulin-binding transcription activator 1 (CAMTA 1) and auxininduced gene X15 and SAUR32 are involved in auxin-mediated regulatory processes (Figure 6A). Similarly, some of these genes such as CAMTA 1 [41,42] and inositol-3-phosphate synthase $[43,44]$ were identified to function in response to drought or salt stress. On the other hand, a few DEGs, such as Ethylene receptor 2 and ethylene responsive factors (ERFs) including ERF04, ERF05, ERF09, ERF12, RAP2-3, RAP2-10, ERF11, ERF104, TEM1 and $T O E 3$, are involved in ethylene signaling pathway (Figure 6C). Inducible expression of $R A P 2-3$, as well as RAP2-12 and RAP2-2, confers tolerance to anoxia, oxidative and osmotic stresses [45]. The ERF6 overexpressing transgenic lines are hypersensitive to osmotic stress, while the growth of erf5erf6 loss-of-function mutants is less affected by stress [46]. These results demonstrated that the overexpression of bolTLP1 significantly affects the expression levels of a set of genes involved in the ABA, ethylene and auxin signaling pathways. Moreover. The homologues of a large proportion of these genes have been demonstrated to function in defending against abiotic stress in diverse plants. Our results also support the role of these genes in the bolTLP1-mediated tolerance to salt and drought stresses. This finding provided new insight into the roles of phytohormone-associated genes in response to abiotic stress in broccoli. Moreover, beside RD2 and RD22, three other proteins VOZ2, LSM1B and MDH were confirmed to directly interact with bolTLP1, and their genes displayed differently expressed pattens between the 35S::bolTLP1 broccoli and vector controls (Figure 8). The investigations demonstrated that VOZ transcription factors act as positive regulators of salt tolerance in Arabidopsis [47]. LSM1-7 are the components of the $5^{\prime}-3^{\prime}$ mRNA decay pathway, which play a crucial role in regulating ABA signaling and modulating ABA-dependent expression of stress related transcription factors from the AP2/ERF/DREB family [48]. Overexpression of plastidic maize NADP-malate dehydrogenase ( $Z m N A D P-M D H)$ increases tolerance to salt stress in Arabidopsis [49]. It suggested that these three abotic-stress associated genes could also play important roles in bolTLP1-mediated regulatory network.

Another remarkably overrepresented group of DEGs was associated with various enzymes, particularly proteolysis, hydrolase and oxidoreductase. The DEGs involved in proteolysis and hydrolase activity mainly function as lectin and kunitz-type protease inhibitors such as serine protease inhibitor, cysteine proteinase inhibitor and trypsin inhibitor (Figure 6E). Plant lectins and protease inhibitors constitute a class of proteins which play a crucial role in plant defense against insect and pathogen attacks [50,51]. Previous investigations confirmed that protease inhibitors also function in abiotic stress. The constitutive expression of a trypsin protease inhibitor confers tolerance to multiple stresses in transgenic tobacco [52]. Oryzacystatin-I (OCI) is a rice cysteine proteinase inhibitor. Ectopic overexpression of OCI can enhance drought stress tolerance in soybean and Arabidopsis [53]. AtCYSa and AtCYSb are two cysteine proteinase inhibitors from Arabidopsis. Overexpression of these inhibitors in transgenic yeast and Arabidopsis increases their resistance to high salt, drought, oxidative and cold stresses [54]. The present study confirmed that all these DEGs involved in proteolysis and hydrolase activity displayed significantly up-regulated expression in 35S::bolTLP1 broccoli (Figure 6E). These findings indicated that the increased expression levels of these genes could also play important roles in promoting salt and drought tolerance of the bolTLP1 transgenic plants. 
A series of histone variants including H1.1, H2A.1, H2A.2, H2A.5, H2B.3, H2A.7, $H 2 B .8$ and DDM1 were also significantly overrepresented in the DEGs (Figure 6F). These genes are important epigenetic regulators, and display significantly higher expression levels in the 35S::bolTLP1 broccoli than those in the vector controls (Figure 6F). TaH2A.7 in wheat can enhance drought tolerance and promote stomatal closure when overexpressed in Arabidopsis [55]. In Arabidopsis, DDM1 is an epigenetic link between salicylic acid metabolism and heterosis, in which salicylic acid can protect plants from pathogens and abiotic stress [56]. Consistently, the mutants of DDM1 show higher sensitivity to $\mathrm{NaCl}$ stress than the wild type plants [57]. In addition, the data confirmed that over 60 genes involved in glycosinolate and sulfur compound synthetic and metabolic processes showed significant differential expression patterns in the bolTLP1 overexpressing transgenic plants and the vector controls. Genes associated with sulfur compound synthesis were up-regulated expression in 35S::bolTLP1 broccoli (Figure 6D). Sulfur compound such as glucosinolates are best known for their roles in plant defense against herbivores and pathogens as well as their cancer-preventive properties [58-60]. A few investigations confirmed that glucosinolates also contributed to abiotic stress [61-64]. These results suggested that the histone variants and these sulfur compound-associated genes may also play an important role in bolTLP1mediated tolerance to abiotic stress.

\section{Materials and Methods}

\subsection{Plant Materials and Stress Treatment}

Homozygous broccoli seeds of KRJ-012 were kindly provided by Dr. Hanmin Jiang, Tianjin Kernel Vegetable Research Institute, Tianjin, China. The seeds were planted in a greenhouse with a $16 \mathrm{~h} / 8 \mathrm{~h}$ light/dark cycle at $25^{\circ} \mathrm{C}$ and $22^{\circ} \mathrm{C}$, respectively. The 25 -dayold seedlings were subjected to salt and drought stresses. For the salt-stress treatment, at least 20 individual plants were irrigated with $200 \mathrm{mM} \mathrm{NaCl}$. To mimic the drought stress, 30 individual plants were irrigated with $300 \mathrm{mM}$ mannitol. The leaves of each plant were harvested at $0,4,8$ and $24 \mathrm{~h}$ after these treatments, frozen immediately in liquid nitrogen, and then stored at $-80{ }^{\circ} \mathrm{C}$. To evaluate the salt tolerance of 35S::bolTLP1 broccoli, at least 18 individual plants from three independent transgenic lines were continually irrigated with $200 \mathrm{mM} \mathrm{NaCl}$ (Tianjin Kemiou Chemical Reagent Co., Ltd., Tianjin, China), and three such replicated treatments were conducted. Similarly, a total of 48 individual plants from three independent 35S:::bolTLP1 broccoli lines were divided into three groups, and unirrigated to evaluate their drought tolerance. In addition, at least three 35S::bolTLP1 Arabidopsis lines in $1 / 2$ MS medium or in soil were treated with 125 and $200 \mathrm{mM} \mathrm{NaCl}$ to mimic salt stress. Three 35S::bolTLP1 Arabidopsis lines in 1/2 MS medium were treated with 200 and $300 \mathrm{mM}$ mannitol to mimic drought stress. The 35S::bolTLP1 Arabidopsis planted in soil were also unirrigated to further evaluate their drought tolerance. To validate the reliability of the assessments of salt and drought tolerance in 35S::bolTLP1 Arabidopsis, three replicates were performed for each treatment.

\subsection{BolTLP1 Cloning and Phylogenetic Analysis}

The ESTs of bolTLP1 were identified from the broccoli transcriptome data, and the specific primers (bolTLP1-F/bolTLP1-R) were designed to amplify the full coding sequences of bolTLP1 based on these expressed sequence tags (ESTs) (Table S5). Total RNAs were isolated from the 25-day-old broccoli seedlings by using TRIzol reagent (Invitrogen, Carlsbad, CA, USA) according to the manufacturer's instructions. The first-strand cDNA was then synthesized using M-MLV reverse transcriptase (Promega, Madison, WI, USA), and the coding sequences of bolTLP1 were amplified by PCR. Because the genome data of broccoli is still limited, to elucidate the sequence homology of bolTLP1 with other TLP genes, those genes containing a TLP domain were identified from the genome database of Brassica oleracea var. oleracea, which is genetically closely related with broccoli (Table S6). The deduced amino acid sequences of bolTLP1 and its homologous genes from B. oleracea var. oleracea were used to construct the phylogenetic tree using the neighbor-joining method 
by the MEGA 6.0 program with the following parameters: bootstrap value of 1000, poisson correction and pairwise deletion [65].

\subsection{BolTLP1 Expression Profile Analysis}

Total RNAs from the leaves of individual plants, which were harvested at different time points after imposing salt or drought stress, were isolated and reverse transcribed to cDNAs. Specific primers of bolTLP1 (qbolTLP1-F/qbolTLP1-R) was designed by Primer premier 5.0 software (Table S5). The bolActin gene from broccoli was selected as the internal control and Faststart Universal SYBR Green Master (Roche, Basel, Switzerland) was used in quantitative real-time RT-PCR (qRT-PCR) assay. The relative expression levels of bolTLP1 under salt or drought stress were calculated by the comparative $2^{-\Delta \Delta C T}$ method. Three biological replicates and three technological replicates were performed to ensure the reliability of quantitative analysis.

\subsection{Expression Vector Construction and Genetic Transformation}

The full coding sequence of bolTLP1 with incorporated Nco I and BstE II restriction sites was amplified (Table S5) and cloned into the pEASY-T1 vector. The insert released from the pEASY-T1 vector with Nco I/BstE II double digestion was sub-cloned into the pCAMBIA3301 binary vector. The recombinant 35S::bolTLP1 plasmids and vectors without exogenous gene insertion (empty vectors) were transformed into A. tumefaciens strain LBA4404. Agrobacterium-mediated Arabidopsis genetic transformation was performed via the floral dip method. The transgenic Arabidopsis lines were screened by spraying 1:10,000 dilute Basta solution and further identified by PCR using specific primers (bolTLP1F/bolTLP1-R) and combined primers (35S-F/bolTLP1-R). Simultaneously, the plants with empty vector were identified by PCR using specific primer pair (35S-F/NOS-R). The transgenic Arabidopsis plants were further verified by qRT-PCR using unique primers (qbolTLP1-F/qbolTLP1-R) and atActin as an internal control (Table S5). The 35S::bolTLP1 plasmids and empty vectors were also transformed into broccoli using Agrobacteriummediated method. In brief, broccoli seeds were rinsed with 75\% ethanol for $2 \mathrm{~min} ; 2 \%$ $\mathrm{NaClO}$ (Tianjin Fengchuan Chemical Reagent Co., Ltd., Tianjin, China) was then further used for surface sterilization of the seeds for $10 \mathrm{~min}$. The sterilized seeds were planted on Murashige and Skoog (MS) medium with a $16 \mathrm{~h} / 8 \mathrm{~h}$ light/dark cycle at $22{ }^{\circ} \mathrm{C}$. The hypocotyls of 7-day-old broccoli seedlings were cut to $0.5 \mathrm{~cm}$ and pre-cultured on MS medium containing $1.5 \mathrm{mg} / \mathrm{L}$ 6-BA and $0.15 \mathrm{mg} / \mathrm{L}$ NAA for 2 days. Then, the truncated hypocotyls were dipped into a suspension of Agrobacterium containing the 35S::bolTLP1 expression vector or an empty vector for $1 \mathrm{~min}$. The infected explants were transferred onto co-cultivation medium with $1 \mathrm{mg} / \mathrm{L}$ 6-BA, $0.1 \mathrm{mg} / \mathrm{L}$ NAA and $100 \mu \mathrm{mol} / \mathrm{L}$ acetosyringone, and cultured for 2 days in the dark. Subsequently, the explants were washed with sterile water and transferred onto MS medium containing $1.6 \mathrm{mg} / \mathrm{L}$ 6-BA, $0.2 \mathrm{mg} / \mathrm{L}$ NAA and $200 \mathrm{mg} / \mathrm{L}$ cefotaxime for 10 days for callus induction. The differentiated explants were transferred into screening medium supplemented with $3 \mathrm{mg} / \mathrm{L}$ Basta for 2 weeks. Finally, buds that continued to differentiate were transferred to the $1 / 2 \mathrm{MS}$ medium containing $1 \mathrm{mg} / \mathrm{L}$ IBA for root initiation. The transgenic broccoli plants were further verified by PCR using prime pair (35S-F/ bolTLP1-R) and qRT-PCR using unique primers (qbolTLP1F/qbolTLP1-R) (Table S5).

\subsection{Transcriptome Sequencing and Data Analysis}

Leaves from six 30-day-old individual plants of per independent 35S::bolTLP1 broccoli line were equally mixed and used to perform three batches of independent RNA isolation. Equal amounts of RNA from each independent 35S::bolTLP1 broccoli line were then used to construct sequencing library. In total, two such sequencing libraries from two independent 35S:: bolTLP1 broccoli lines and one sequencing library from the vector controls were constructed. The sequencing reaction was conducted by the Illumina HiSeq ${ }^{\mathrm{TM}} 2500$ sequencing platform (Beijing Genomics Institute, Shenzhen, China) with three technolog- 
ical replicates. The clean reads were annotated and mapped to the reference genome of B. oleracea var. oleracea. The expression levels of genes were calculated by the fragments per kilobase of transcript sequence per million base pairs sequenced (FPKM). The significantly expression levels of genes between the 35S::bolTLP1 broccoli and the vector controls were identified based on the thresholds: $\mid \log _{2}$ (fold-change (35S:: bolTLP1 broccoli/vector control) $) \mid>1$ and corrected $p$-value $<0.05$. Gene Ontology (GO) analysis of the differentially expressed genes (DEGs) was performed by the agriGO platform. Available online: http:/ /bioinfo.cau.edu.cn/agriGO/ (accessed on 4 May 2018).

\subsection{Differentially Expressed Gene Identification by $q R T-P C R$}

The gene expression profiles detected by comparative transcriptome analysis were verified by qRT-PCR by using the specific primer pairs (Table S5). Three independent 35S:::bolTLP1 broccoli lines (Line 3, Line 5 and Line 9) were used to perform three biological replicates. Similar to RNA isolation in transcriptome sequencing, RNAs from leaves of six randomly selected 30-day-old plants per 35S::bolTLP1 broccoli line were isolated and reverse transcribed to cDNAs. The comparative $2^{-\Delta \Delta C T}$ method was conducted to calculate the relative expression levels of genes using bolActin as an internal control. To further ensure the reliability of qRT-PCR, three technological replicates were carried out.

\subsection{Yeast Two-Hybrid Screening and Assays}

The proteins which interact with bolTLP1 were screened using Matchmaker ${ }^{\mathrm{TM}}$ Gold Yeast Two-Hybrid System (Clontech, Mountain View, CA, USA) according to the manufacturer's instructions. In brief, the full-length coding sequences of bolTLP1 with the $\mathrm{Nco}$ I and BamH I restriction enzyme sites were constructed into the bait vector pGBKT7 (Table S5). The recombinant bait vector pGBKT7-bolTLP1 was transformed into the $\mathrm{Y} 2 \mathrm{H}$ Gold yeast train, and the autoactivation and toxicity of bolTLP1 were detected. The positive pGBKT7-bolTLP1 yeast then was mixed and mated with the universal Arabidopsis Mate \& Plate library (Clontech, Mountain View, CA, USA). The positive mating yeast was screened on SD/-Trp-Leu-His-Ade medium with $0.5 \mathrm{mM}$ 3-Amino-1, 2, 4-triazole (3-AT), which could effectively inhibit the autoactivation of bolTLP1. The plasmids from the mating yeast were isolated and used as a template to amplify the candidate genes using the universal primers (T7-F/3'AD-R) (Table S5). To further confirm the relationship of bolTLP1 and its candidate interacting proteins, the broccoli homologs of the positive Arabidopsis genes were cloned and inserted into the bait vector pGBKT7. In this case, bolTLP1 was inserted into the prey vector pGADT7 (Table S5). Subsequently, the yeast two-hybrid experiments were conducted according to the manufacturer's instructions. Transcript expression levels of the broccoli genes encoding proteins interacting with bolTLP1 were analyzed by qRT-PCR as mentioned above (Table S5).

\section{Conclusions}

Overexpression of bolTLP1 significantly increased the salt and drought tolerance in both Arabidopsis and broccoli. BolTLP1 may directly interact with stress responseassociated proteins RD2, RD22, VOZ2, LSM1B and MDH to regulate a series of genes involved in phytohormone (ABA, ethylene and auxin)-mediated signaling pathways, hydrolase/oxidoreductase activity, sulfur compound synthesis, and histone variants, which could play important roles in bolTLP1-mediated tolerance to salt and drought stresses in broccoli (Figure 9). BolTLP1 is a potential candidate gene in breeding crops with high tolerance to abiotic stress via genetic engineering. 


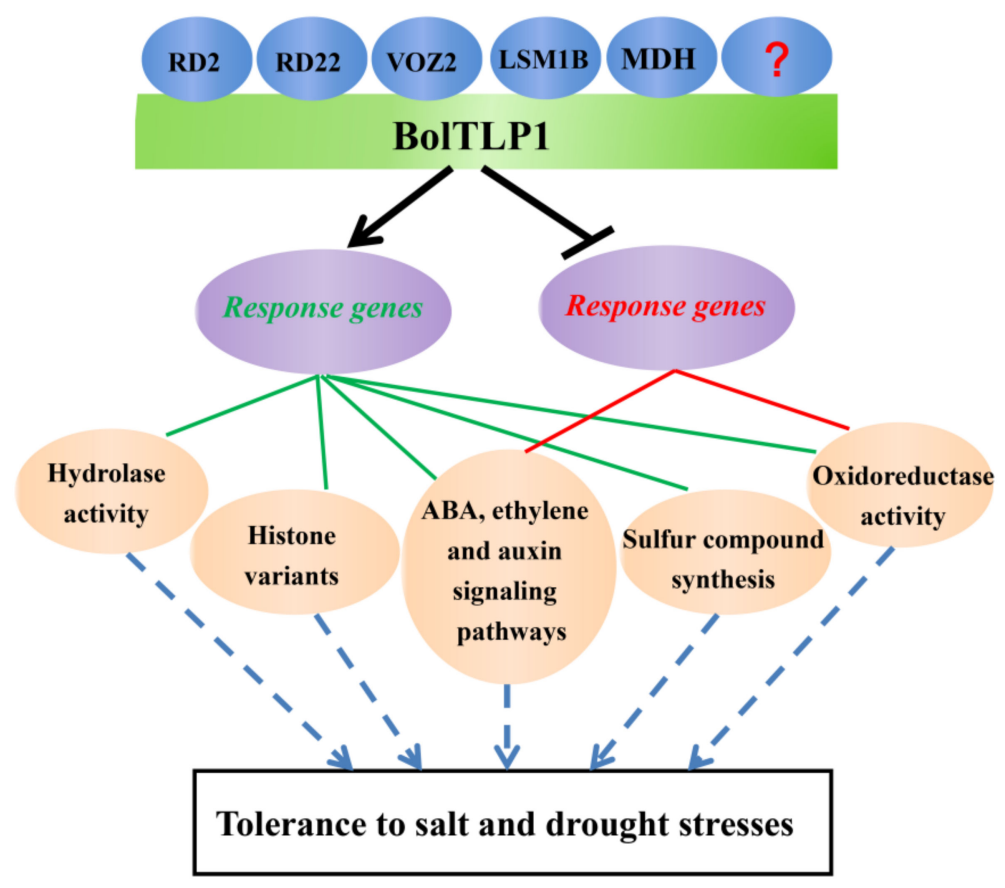

Figure 9. A proposed model indicating the regulatory processes of bolTLP1 in increasing the tolerance to salt and drought stresses in broccoli. This model indicated that in response to salt and drought stresses, bolTLP1 may directly interact with RD2, RD22, VOZ2, LSM1B and MDH to positively regulate a series of genes involved in phytohormone (ABA, ethylene and auxin)-mediated signaling pathways, hydrolase activity, oxidoreductase activity, sulfur compound synthesis and histone variants. Meanwhile, the expression of several genes involved in phytohormone (ABA, ethylene and auxin)-mediated signaling pathways and oxidoreductase activity were inhibited by the overexpression of bolTLP1. The green font and lines showed the positive regulation of response genes. The red font and lines showed the negative regulation of response genes.

Supplementary Materials: The following are available online at https:/ /www.mdpi.com/article/10 $.3390 / \mathrm{ijms} 222011132 / \mathrm{s} 1$.

Author Contributions: L.L. and L.H. performed the molecular biological experiments in Arabidopsis and broccoli; Y.Z. and Y.P. performed the data analysis; X.Z., X.H. and M.L. performed the yeast twohybrid assay; C.C. and Y.Z. conducted the phenotypic assay; H.L. and C.W. designed the project and wrote the manuscript. All authors have read and agreed to the published version of the manuscript.

Funding: This work was funded by grants from the Natural Science Foundation of China (No. 31872115 and No. 31470669), the State Key Laboratory of Tree Genetics and Breeding, Northeast Forestry University, China (NFUZD2015), the National science and technology major special project of transgenes (2018ZX08020003-001-003) and the Science and Technology of Tianjin, China (No. 18ZXZYNC00160). The funding body was not involved in the design of the study and analysis or interpretation of the data and in writing of the manuscript.

Institutional Review Board Statement: Not applicable.

Informed Consent Statement: Not applicable.

Data Availability Statement: Data are contained in Supplementary Materials.

Acknowledgments: We are grateful to Hanmin Jiang of Tianjin Kernel Vegetable Research Institute, Tianjin, China, for kindly providing the homozygous broccoli seeds.

Conflicts of Interest: The authors declare no competing interests. 


\section{References}

1. Liu, J.J.; Sturrock, R.; Ekramoddoullah, A.K. The superfamily of thaumatin-like proteins: Its origin, evolution, and expression towards biological function. Plant Cell Rep. 2010, 29, 419-436. [CrossRef] [PubMed]

2. Cornelissen, B.J.; Hooft, V.; Bol, J.F. A tobacco mosaic virus-induced tobacco protein is homologous to the sweet-testing protein thaumatin. Nature 1986, 32, 1531-1532.

3. Ghosh, R.; Chakrabarti, C. Crystal structure analysis of NP24-I, A thaumatin-like protein. Planta 2008, 228, 883-890. [CrossRef]

4. Petre, B.; Major, I.; Rouhier, N.; Duplessis, S. Genome-wide analysis of eukaryote thaumatin-like proteins (TLPs) with an emphasis on poplar. BMC Plant Biol. 2011, 11, 33. [CrossRef] [PubMed]

5. Christensen, A.B.; Cho, B.H.; Næsby, M.; Gregersen, P.L.; Brandt, J.; Madriz-Ordeñana, K.; Collinge, D.B.; Thordal-Christensen, H. The molecular characterization of two barley proteins establishes the novel PR-17 family of pathogenesis-related proteins. Mol. Plant Pathol. 2002, 3, 135-144. [CrossRef] [PubMed]

6. Van Loon, L.C.; Rep, M.; Pieterse, C.M.J. Significance of inducible defense-related proteins in infected plants. Annu. Rev. Phytopathol. 2006, 44, 135-162. [CrossRef]

7. Iqbal, I.; Tripathi, R.K.; Wilkins, O.; Singh, J. Thaumatin-Like Protein (TLP) gene family in barley: Genome-wide exploration and expression analysis during germination. Genes 2020, 11, 1080. [CrossRef] [PubMed]

8. Ye, X.Y.; Wang, H.X.; Ng, T.B. First chromatographic isolation of an antifungal thaumatin-like protein from French bean legumes and demonstration of its antifungal activity. Biochem. Biophys. Res. Commun. 1999, 263, 130-134. [CrossRef]

9. Vitali, A.; Pacini, L.; Bordi, E.; De Mori, P.; Pucillo, L.; Maras, B.; Botta, B.; Brancaccio, A.; Giardina, B. Purification and characterization of an antifungal thaumatin-like protein from Cassia didymobotrya cell culture. Plant Physiol. Biochem. 2006, 44, 604-610. [CrossRef]

10. Zhang, J.; Wang, F.; Liang, F.; Zhang, Y.; Ma, L.; Wang, H.; Liu, D. Functional analysis of a pathogenesis-related thaumatin-like protein gene TaLr35PR5 from wheat induced by leaf rust fungus. BMC Plant Biol. 2018, 18, 76. [CrossRef]

11. Krebitz, M.; Wagner, B.; Ferreira, F.; Peterbauer, C.; Campillo, N.; Witty, M.; Kolarich, D.; Steinkellner, H.; Scheiner, O.; Breiteneder, H. Plant-based heterologous expression of Mald2, a thaumatin-like protein and allergen of apple (Malus domestica), and its characterization as an antifungal protein. J. Mol. Biol. 2003, 329, 721-730. [CrossRef]

12. Liu, D.Q.; He, X.; Li, W.X.; Chen, C.Y.; Ge, F. Molecular cloning of a thaumatin-like protein gene from Pyrus pyrifolia and overexpression of this gene in tobacco increased resistance to pathogenic fungi. Plant Cell Tissue Organ Cult. 2012, 111, 29-39. [CrossRef]

13. Singh, N.K.; Kumar, K.R.; Kumar, D.; Shukla, P.; Kirti, P.B. Characterization of a pathogen induced thaumatin-like protein gene AdTLP from Arachis diogoi, a wild peanut. PLoS ONE 2013, 8, e83963. [CrossRef] [PubMed]

14. Aghazadeh, R.; Zamani, M.; Motallebi, M.; Moradyar, M. Agrobacterium-mediated transformation of the Oryza sativa thaumatinlike protein to canola (R Line Hyola308) for enhancing resistance to Sclerotinia sclerotiorum. Iran. J. Biotechnol. 2017, 15, 201-207. [CrossRef] [PubMed]

15. Chen, W.P.; Chen, P.D.; Liu, D.J.; Kynast, R.; Friebe, B.; Velazhahan, R.; Muthukrishnan, S.; Gil, B.S. Development of wheat scab symptoms is delayed in transgenic wheat plants that constitutively express a rice thaumatin-like protein gene. Theor. Appl. Genet. 1999, 99, 755-760. [CrossRef]

16. Mahdavi, F.; Sariah, M.; Maziah, M. Expression of rice thaumatin-like protein gene in transgenic banana plants enhances resistance to fusarium wilt. Appl. Biochem. Biotechnol. 2012, 166, 1008-1019. [CrossRef]

17. Misra, R.C.; Sandeep; Kamthan, M.; Kumar, S.; Ghosh, S. A thaumatin-like protein of Ocimum basilicum confers tolerance to fungal pathogen and abiotic stress in transgenic Arabidopsis. Sci. Rep. 2016, 6, 25340. [CrossRef]

18. Jung, Y.C.; Lee, H.J.; Yum, S.S.; Soh, W.Y.; Cho, D.Y.; Auh, C.K.; Lee, T.K.; Soh, H.C.; Kim, Y.S.; Lee, S.C. Drought-inducible-but ABA-independent-thaumatin-like protein from carrot (Daucus carota L.). Plant Cell Rep. 2005, 24, 366-373. [CrossRef]

19. Muoki, R.C.; Paul, A.; Kumar, S. A shared response of thaumatin like protein, chitinase, and late embryogenesis abundant protein 3 to environmental stresses in tea Camellia sinensis (L.) O. Kuntze. Funct. Integr. Genom. 2012, 12, 565-571. [CrossRef] [PubMed]

20. Salzman, R.A.; Tikhonova, I.; Bordelon, B.P.; Hasegawa, P.M.; Bressan, R.A. Coordinate accumulation of antifungal proteins and hexoses constitutes a developmentally controlled defense response during fruit ripening in grape. Plant Physiol. 1998, 117, 465-472. [CrossRef]

21. Sakamoto, Y.; Watanabe, H.; Nagai, M.; Nakade, K.; Takahashi, M.; Sato, T. Lentinula edodes tlg1 encodes a thaumatin-like protein that is involved in lentinan degradation and fruiting body senescence. Plant Physiol. 2006, 141, 793-801. [CrossRef]

22. Damme, E.; Charels, D.; Menu-Bouaouiche, L.; Proost, P.; Barre, A.; Rougé, P.; Peumans, W.J. Biochemical, molecular and structural analysis of multiple thaumatin-like proteins from the elderberry tree (Sambucus nigra L.). Planta 2002, 214, 853-862. [CrossRef]

23. Skadsen, R.W.; Sathish, P.; Kaeppler, H.F. Expression of thaumatin-like permatin PR-5 genes switches from the ovary wall to the aleurone in developing barley and oat seeds. Plant Sci. 2000, 156, 11-22. [CrossRef]

24. Singh, S.; Tripathi, R.K.; Lemaux, P.G.; Buchanan, B.B.; Singh, J. Redox-dependent interaction between thaumatin-like protein and $\beta$-glucan influences malting quality of barley. Proc. Natl. Acad. Sci. USA 2017, 114, 7725-7730. [CrossRef] [PubMed]

25. Ilahy, R.; Tlili, I.; Pék, Z.; Montefusco, A.; Siddiqui, M.W.; Homa, F.; Hdider, C.; R'Him, T.; Lajos, H.; Lenucci, M.S. Pre- and Post-harvest Factors Affecting Glucosinolate Content in Broccoli. Front. Nutr. 2020, 7, 147. [CrossRef]

26. Zhu, J.K. Abiotic Stress Signaling and Responses in Plants. Cell 2016, 167, 313-324. [CrossRef] [PubMed] 
27. Venegas-Molina, J.; Proietti, S.; Pollier, J.; Orozco-Freire, W.; Ramirez-Villacis, D.; Leon-Reyes, A. Induced tolerance to abiotic and biotic stresses of broccoli and Arabidopsis after treatment with elicitor molecules. Sci. Rep. 2020, 10, 10319. [CrossRef] [PubMed]

28. Chevilly, S.; Dolz-Edo, L.; López-Nicolás, J.M.; Morcillo, L.; Vilagrosa, A.; Yenush, L.; Mulet, J.M. Physiological and molecular characterization of the differential response of broccoli (Brassica oleracea var. Italica) cultivars reveals limiting factors for broccoli tolerance to drought stress. J. Agric. Food Chem. 2021, 69, 10394-10404. [CrossRef] [PubMed]

29. Tian, Y.; Tian, Y.; Luo, X.; Zhou, T.; Huang, Z.; Liu, Y.; Qiu, Y.; Hou, B.; Sun, D.; Deng, H.; et al. Identification and characterization of microRNAs related to salt stress in broccoli, using high-throughput sequencing and bioinformatics analysis. BMC Plant Biol. 2014, 14, 226. [CrossRef]

30. Li, H.; Wang, Y.; Wu, M.; Li, L.H.; Li, C.; Han, Z.P.; Yuan, J.Y.; Chen, C.B.; Song, W.Q.; Wang, C.G. Genome-wide identification of AP2/ERF transcription factors in cauliflower and expression profiling of the erf family under salt and drought stresses. Front. Plant Sci. 2017, 8, 946. [CrossRef]

31. Muthusamy, M.; Kim, J.Y.; Yoon, E.K.; Kim, J.A.; Lee, S.I. BrEXLB1, a Brassica rapa expansin-like B1 gene is associated with root development, drought stress response, and seed germination. Genes 2020, 11, 404. [CrossRef]

32. Zhao, B.Y.; Hu, Y.F.; Li, J.J.; Yao, X.; Liu, K.D. BnaABF2, a bZIP transcription factor from rapeseed (Brassica napus L.), enhances drought and salt tolerance in transgenic Arabidopsis. Bot. Stud. 2016, 57, 12. [CrossRef] [PubMed]

33. Wang, M.; Yuan, F.; Hao, H.; Zhang, Y.; Zhao, H.; Guo, A.; Hu, J.; Zhou, X.; Xie, C.G. BolOST1, an ortholog of Open Stomata 1 with alternative splicing products in Brassica oleracea, positively modulates drought responses in plants. Biochem. Biophys. Res. Commun. 2013, 442, 214-220. [CrossRef] [PubMed]

34. Samota, M.K.; Sasi, M.; Awana, M.; Yadav, O.P.; Amitha Mithra, S.V.; Tyagi, A.; Kumar, S.; Singh, A. Elicitor-induced biochemical and molecular manifestations to improve drought tolerance in rice (Oryza Sativa, L.) through seed-priming. Front. Plant Sci. 2017, 8, 934. [CrossRef] [PubMed]

35. Harshavardhan, V.T.; Van Son, L.; Seiler, C.; Junker, A.; Weigelt-Fischer, K.; Klukas, C.; Altmann, T.; Sreenivasulu, N.; Bäumlein, H.; Kuhlmann, M. AtRD22 and AtUSPL1, members of the plant-specific BURP domain family involved in Arabidopsis thaliana drought tolerance. PLoS ONE 2014, 9, e110065. [CrossRef]

36. Ijaz, R.; Ejaz, J.; Gao, S.; Liu, T.; Imtiaz, M.; Ye, Z.; Wang, T. Overexpression of annexin gene AnnSp2, enhances drought and salt tolerance through modulation of ABA synthesis and scavenging ROS in tomato. Sci. Rep. 2017, 7, 12087. [CrossRef]

37. Luo, X.; Bai, X.; Sun, X.; Zhu, D.; Liu, B.; Ji, W.; Cai, H.; Cao, L.; Wu, J.; Hu, M.; et al. Expression of wild soybean WRKY20 in Arabidopsis enhances drought tolerance and regulates ABA signaling. J. Exp. Bot. 2013, 64, 2155-2169. [CrossRef]

38. Takeuchi, J.; Okamoto, M.; Mega, R.; Kanno, Y.; Ohnishi, T.; Seo, M.; Todoroki, Y. Abscinazole-E3M, a practical inhibitor of abscisic acid 8'-hydroxylase for improving drought tolerance. Sci. Rep. 2016, 6, 37060. [CrossRef]

39. Ju, Y.L.; Yue, X.F.; Min, Z.; Wang, X.H.; Fang, Y.L.; Zhang, J.X. VvNAC17, a novel stress-responsive grapevine (Vitis vinifera L.) NAC transcription factor, increases sensitivity to abscisic acid and enhances salinity, freezing, and drought tolerance in transgenic Arabidopsis. Plant Physiol. Biochem. 2020, 146, 98-111. [CrossRef]

40. Wang, H.; Zhou, L.; Fu, Y.; Cheung, M.Y.; Wong, F.L.; Phang, T.H.; Sun, Z.; Lam, H.M. Expression of an apoplast-localized BURP-domain protein from soybean (GmRD22) enhances tolerance towards abiotic stress. Plant Cell Environ. 2012, 35, 1932-1947. [CrossRef]

41. Galon, Y.; Aloni, R.; Nachmias, D.; Snir, O.; Feldmesser, E.; Scrase-Field, S.; Boyce, J.M.; Bouché, N.; Knight, M.R.; Fromm, H. Calmodulin-binding transcription activator 1 mediates auxin signaling and responds to stresses in Arabidopsis. Planta 2010, 232, 165-178. [CrossRef]

42. Pandey, N.; Ranjan, A.; Pant, P.; Tripathi, R.K.; Ateek, F.; Pandey, H.P.; Patre, U.V.; Sawant, S.V. CAMTA 1 regulates drought responses in Arabidopsis thaliana. BMC Genom. 2013, 14, 216. [CrossRef]

43. Kusuda, H.; Koga, W.; Kusano, M.; Oikawa, A.; Saito, K.; Hirai, M.Y.; Yoshida, K.T. Ectopic expression of myo-inositol 3-phosphate synthase induces a wide range of metabolic changes and confers salt tolerance in rice. Plant Sci. 2015, 232, 49-56. [CrossRef]

44. Das-Chatterjee, A.; Goswami, L.; Maitra, S.; Dastidar, K.G.; Ray, S.; Majumder, A.L. Introgression of a novel salt-tolerant L-myoinositol 1-phosphate synthase from Porteresia coarctata (Roxb.) Tateoka (PcINO1) confers salt tolerance to evolutionary diverse organisms. FEBS. Lett. 2006, 580, 3980-3988. [CrossRef] [PubMed]

45. Papdi, C.; Pérez-Salamó, I.; Joseph, M.P.; Giuntoli, B.; Bögre, L.; Koncz, C.; Szabados, L. The low oxygen, oxidative and osmotic stress responses synergistically act through the ethylene response factor VII genes RAP2.12, RAP2.2 and RAP2.3. Plant J. 2015, 82, 772-784. [CrossRef] [PubMed]

46. Dubois, M.; Skirycz, A.; Claeys, H.; Maleux, K.; Dhondt, S.; De Bodt, S.; Vanden Bossche, R.; De Milde, L.; Yoshizumi, T.; Matsui, M.; et al. Ethylene Response Factor 6 acts as a central regulator of leaf growth under water-limiting conditions in Arabidopsis. Plant Physiol. 2013, 162, 319-332. [CrossRef] [PubMed]

47. Prasad, K.; Xing, D.; Reddy, A. Vascular plant one-zinc-finger (VOZ) transcription factors are positive regulators of salt tolerance in Arabidopsis. Int. J. Mol. Sci. 2018, 19, 3731. [CrossRef]

48. Wawer, I.; Golisz, A.; Sulkowska, A.; Kawa, D.; Kulik, A.; Kufel, J. mRNA Decapping and 5'-3' decay contribute to the regulation of aba signaling in Arabidopsis thaliana. Front. Plant Sci. 2018, 9, 312. [CrossRef]

49. Kandoi, D.; Mohanty, S.; Tripathy, B.C. Overexpression of plastidic maize NADP-malate dehydrogenase (ZmNADP-MDH) in Arabidopsis thaliana confers tolerance to salt stress. Protoplasma 2018, 255, 547-563. [CrossRef] 
50. Koiwa, H.; Bressan, R.A.; Hasegawa, P.M. Regulation of protease inhibitors and plant defense. Trends. Plant. Sci. 1997, 2, 379-384. [CrossRef]

51. Breitenbach Barroso Coelho, L.C.; Marcelino Dos Santos Silva, P.; Felix de Oliveira, W.; de Moura, M.C.; Viana Pontual, E.; Soares Gomes, F.; Guedes Paiva, P.M.; Napoleão, T.H.; Dos Santos Correia, M.T. Lectins as antimicrobial agents. J. Appl. Microbiol. 2018, 125, 1238-1252. [CrossRef]

52. Srinivasan, T.; Kumar, K.R.; Kirti, P.B. Constitutive expression of a trypsin protease inhibitor confers multiple stress tolerance in transgenic tobacco. Plant Cell Physiol. 2009, 50, 541-553. [CrossRef] [PubMed]

53. Quain, M.D.; Makgopa, M.E.; Márquez-García, B.; Comadira, G.; Fernandez-Garcia, N.; Olmos, E.; Schnaubelt, D.; Kunert, K.J.; Foyer, C.H. Ectopic phytocystatin expression leads to enhanced drought stress tolerance in soybean (Glycine max) and Arabidopsis thaliana through effects on strigolactone pathways and can also result in improved seed traits. Plant Biotechnol. J. 2014, 12, 903-913. [CrossRef]

54. Zhang, X.; Liu, S.; Takano, T. Two cysteine proteinase inhibitors from Arabidopsis thaliana, AtCYSa and AtCYSb, increasing the salt, drought, oxidation and cold tolerance. Plant Mol. Biol. 2008, 68, 131-143. [CrossRef]

55. Xu, W.; Li, Y.; Cheng, Z.; Xia, G.; Wang, M. A wheat histone variant gene TaH2A.7 enhances drought tolerance and promotes stomatal closure in Arabidopsis. Plant Cell Rep. 2016, 35, 1853-1862. [CrossRef]

56. Zhang, Q.; Li, Y.; Xu, T.; Srivastava, A.K.; Wang, D.; Zeng, L.; Yang, L.; He, L.; Zhang, H.; Zheng, Z.; et al. The chromatin remodeler DDM1 promotes hybrid vigor by regulating salicylic acid metabolism. Cell Discov. 2016, 2, 16027. [CrossRef]

57. Yao, Y.; Bilichak, A.; Golubov, A.; Kovalchuk, I. $d d m 1$ plants are sensitive to methyl methane sulfonate and $\mathrm{NaCl}$ stresses and are deficient in DNA repair. Plant Cell Rep. 2012, 31, 1549-1561. [CrossRef]

58. Bednarek, P.; Osbourn, A. Plant-microbe interactions: Chemical diversity in plant defense. Science 2009, 324, 746-748. [CrossRef] [PubMed]

59. Clay, N.K.; Adio, A.M.; Denoux, C.; Jander, G.; Ausubel, F.M. Glucosinolate metabolites required for an Arabidopsis innate immune response. Science 2009, 323, 95-101. [CrossRef] [PubMed]

60. Zhang, Y.; Kensler, T.W.; Cho, C.G.; Posner, G.H.; Talalay, P. Anticarcinogenic activities of sulforaphane and structurally related synthetic norbornyl isothiocyanates. Proc. Natl. Acad. Sci. USA 1994, 91, 3147-3150. [CrossRef] [PubMed]

61. Martínez-Ballesta, M.; Moreno-Fernández, D.A.; Castejón, D.; Ochando, C.; Morandini, P.A.; Carvajal, M. The impact of the absence of aliphatic glucosinolates on water transport under salt stress in Arabidopsis thaliana. Front. Plant. Sci. 2015, 6, 524. [CrossRef]

62. Seo, M.S.; Jin, M.; Sohn, S.H.; Kim, J.S. Expression profiles of BrMYB transcription factors related to glucosinolate biosynthesis and stress response in eight subspecies of Brassica rapa. FEBS Open. Bio. 2017, 7, 1646-1659. [CrossRef] [PubMed]

63. Zhao, L.; Wang, C.; Zhu, F.; Li, Y. Mild osmotic stress promotes 4-methoxy indolyl-3-methyl glucosinolate biosynthesis mediated by the MKK9-MPK3/MPK6 cascade in Arabidopsis. Plant Cell Rep. 2017, 36, 543-555. [CrossRef]

64. Martínez-Ballesta, M.D.C.; Moreno, D.A.; Carvajal, M. The physiological importance of glucosinolates on plant response to abiotic stress in Brassica. Int. J. Mol. Sci. 2013, 14, 11607-11625. [CrossRef] [PubMed]

65. Tamura, K.; Stecher, G.; Peterson, D.; Filipski, A.; Kumar, S. MEGA6: Molecular evolutionary genetics analysis version 6.0. Mol. Biol. Evol. 2013, 30, 2725-2729. [CrossRef] [PubMed] 